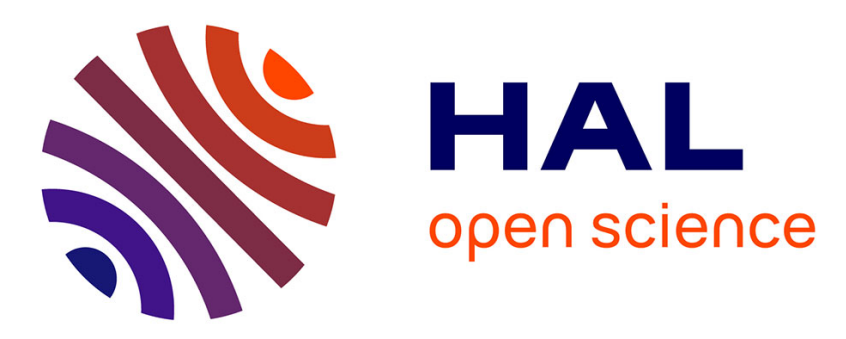

\title{
Effective electrical conductivity of CNT/polymer nanocomposites
}

Xiaoxin Lu, Yu Liu, Lionel Pichon, Delong He, Olivier Dubrunfaut, Jinbo Bai

\section{To cite this version:}

Xiaoxin Lu, Yu Liu, Lionel Pichon, Delong He, Olivier Dubrunfaut, et al.. Effective electrical conductivity of $\mathrm{CNT} /$ polymer nanocomposites. 2020 International Symposium on Electromagnetic Compatibility - EMC EUROPE, Sep 2020, Rome, France. pp.1-4, 10.1109/EMCEUROPE48519.2020.9245805 . hal-03031795

\section{HAL Id: hal-03031795 \\ https://hal.science/hal-03031795}

Submitted on 24 Sep 2021

HAL is a multi-disciplinary open access archive for the deposit and dissemination of scientific research documents, whether they are published or not. The documents may come from teaching and research institutions in France or abroad, or from public or private research centers.
L'archive ouverte pluridisciplinaire HAL, est destinée au dépôt et à la diffusion de documents scientifiques de niveau recherche, publiés ou non, émanant des établissements d'enseignement et de recherche français ou étrangers, des laboratoires publics ou privés. 


\section{Effective electrical conductivity of CNT/polymer nanocomposites}

\author{
Xiaoxin $\mathrm{Lu}$ \\ Université Paris-Saclay, \\ CentraleSupélec, CNRS, Laboratoire \\ de Génie Electrique et Electronique de \\ Paris, 91192, Gif-sur-Yvette, France. \\ Sorbonne Université, CNRS, \\ Laboratoire de Génie Electrique et \\ Electronique de Paris, 75252, Paris, \\ France \\ Delong He \\ Université Paris-Saclay, \\ CentraleSupélec, CNRS, Laboratoire \\ Mécanique des Sols, Structures et \\ Matériaux, 91190, Gif-sur-Yvette, \\ France \\ delong.he@centralesupelec.fr
}

\author{
Yu Liu \\ Université Paris-Saclay, \\ CentraleSupélec, CNRS, Laboratoire \\ Mécanique des Sols, Structures et \\ Matériaux, 91190, Gif-sur-Yvette, \\ France.
}

Olivier Dubrunfaut Université Paris-Saclay, CentraleSupélec, CNRS, Laboratoire de Génie Electrique et Electronique de Paris, 91192, Gif-sur-Yvette, France. Sorbonne Université, CNRS, Laboratoire de Génie Electrique et Electronique de Paris, 75252, Paris, France

olivier.dubrunfaut@centralesupelec.fr

\author{
Lionel Pichon \\ Université Paris-Saclay, \\ CentraleSupélec, CNRS, Laboratoire \\ de Génie Electrique et Electronique de \\ Paris, 91192, Gif-sur-Yvette, France. \\ Sorbonne Université, CNRS, \\ Laboratoire de Génie Electrique et \\ Electronique de Paris, 75252, Paris, \\ France \\ lionel.pichon@centralesupelec.fr
}

\author{
Jinbo Bai \\ Université Paris-Saclay, \\ CentraleSupélec, CNRS, Laboratoire \\ Mécanique des Sols, Structures et \\ Matériaux, 91190, Gif-sur-Yvette, \\ France \\ jinbo.bai@centralesupelec.fr
}

\begin{abstract}
This paper studies the electromagnetic behavior of carbon nanotubes (CNTs) based nanocomposites. Such nanocomposites are meaningful for the material design, especially for shielding in EMC applications. A multiscale numerical homogenization method based on a finite element model and incorporating tunneling effect provides is implemented. CNTs are modeled by highly conductive line segments in order to avoid the mesh problems. Effective electrical conductivity is obtained both in statics and time harmonic cases. Numerical predictions are compared to experimental data.
\end{abstract}

Keywords-nanocomposites, electrical conductivity, homogenization, shielding, numerical methods

\section{INTRODUCTION}

In the past decade, carbon nanotubes (CNTs) have been of great interest as filler in polymers due to their excellent electrical and thermal properties, superior mechanical properties, high aspect ratio, and lightweight $[1,2]$. CNT based nanocomposites have demonstrated numerous extraordinary properties, such as high electrical conductivity, low percolation threshold, and good dielectric and mechanical properties [3,5]. Their electromagnetic properties also correspond to EMC and shielding applications [6,7]. It has been reported that the effective electrical conductivity of CNT enhances polymer composites obeys percolation-like power law attributed to the formation of conductive CNT networks, which is dominated by tunneling effect after percolation threshold [6,7]. Tunneling effect is a quantum phenomenon that allows the electric conduction across small isolating barriers between two highly conducting fillers. When the characteristic distance between CNTs is at nanometric scale, the corresponding tunneling current plays an important role in the generation of the current path together with the conductive CNTs, leading to an increase of several orders of magnitude of the electrical conductivity at extremely low volume fractions of CNTs denoting the percolation threshold [8-10].
Beside experimental studies and for a better understanding of the electrical phenomenon and the design purpose of smart structural applications, both analytical and numerical studies are still on going. Analytical approaches show some limitations in the quantitative predictions of electrical properties and in the explanation of local characteristics of the microstructures. Thus, numerical methods are required to better describe the local phenomenon and the effect of material parameters or morphology on the effective electric conductivity. Most of the numerical models are developed based on the statistical resistor network theory and Monte Carlo simulation [11-15].

In this paper a numerical methodology based on finite element method (FEM) is developed to study the effective conductivity of CNT/polymer nanocomposites as well as their percolation threshold. The representative volume element (RVE) containing randomly distributed CNTs are generated, and the FEM simulations taking into account the nonlinear tunneling effect are developed where the distances between CNTs are well described by the introduction of distance function. In addition, the CNTs are modeled by highly conducting line segments in the model according to their high aspect ratio, in order to avoid meshing the thin cylindrical shape. Also, the AC conductivity and the frequencydependent dielectric constants of CNT/polymer was investigated. In both static and dynamic case, the effective conductivity and dielectric constants are evaluated by appropriate definitions of effective quantities through homogenization. Comparisons between measured data and numerical predictions are presented.

\section{MULTISCALE MODELING OF ELECTRICAL BEHAVIOR}

\section{A. Generation of CNT/polymer nanocomposites}

A representative volume element (RVE) is defined in a domain as shown in Fig. 1. The RVE with side length of $\mathrm{L}$ contains N straight CNTs randomly distributed in the polymer 
matrix. The conduction mechanism according to tunneling effect is introduced between neighbouring CNTs when their shortest distance is at nanoscale. In this work, each CNT is assumed to be straight with the length of 1 , and the diameter of $D$. The aspect ratio $\eta=1 / D$ denotes the ratio between the length of CNT and its diameter.

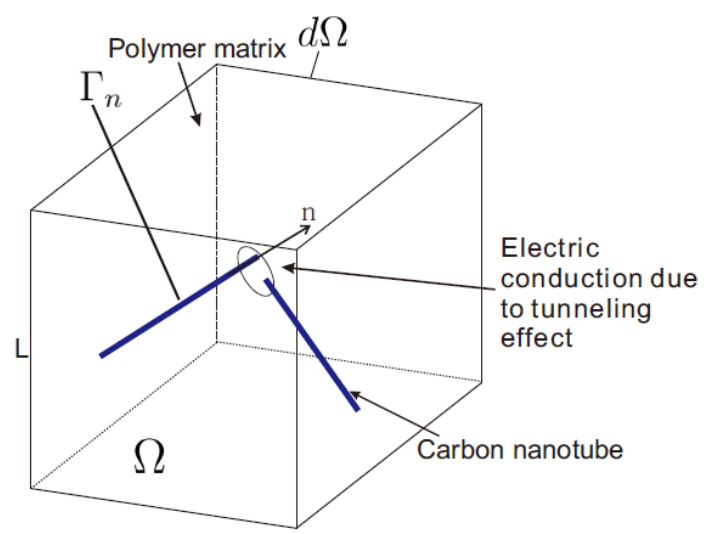

Fig. 1. RVE model of the CNT-polymer nanocomposite.

In this model, the total electric power within the domain $\Omega$, W, which consists of the contribution of both polymer matrix and CNTs, is defined by:

$$
2 W=\int_{\Omega} \omega^{m}(x) d \Omega+\int_{\Gamma} \omega^{c}(x) d \Gamma
$$

where $\Gamma$ denotes collectively the lines associated with CNTs and the density functions of polymer matrix $\left(\omega^{\mathrm{m}}\right)$ and CNTs $\left(\omega^{\mathrm{c}}\right)$, are expressed by:

$$
\omega^{m}(x)=j(x) \cdot E(x) \quad ; \quad \omega^{c}(x)=j^{c}(x) \cdot E^{c}(x)
$$

In the first equation in (2), $E(x)=-\nabla \varphi(x)$ is the electric field, $\mathrm{j}(\mathrm{x})$ is the current density vector and $\varphi$ is the electric potential. Taking into account tunneling effect, it satisfies:

$$
j=\left\{\begin{aligned}
G(E, d) \frac{E}{|E|} & \text { if } d<d_{\text {cutoff }} \\
\sigma^{m} E & \text { if } d \geq d_{\text {cutoff }}
\end{aligned}\right.
$$

where $\sigma^{\mathrm{m}}$ is the electric conductivity tensor of the polymer matrix when neglecting tunneling effect, $\mathrm{d}_{\text {cutoff }}$ is a cut-off distance above which the tunneling effect can be neglected and $\mathrm{G}$ is defined by Simmons [16].

In the second equation in (2), the superscript c denotes line quantities for CNTs, e.g., $\mathrm{j}^{\mathrm{C}}$ is the line current density and $\mathrm{E}^{\mathrm{C}}$ is the line electric field on CNTs which is calculated by taking the gradient of electric potential on the two nodes of line element. CNTs can be considered as rolled-up sheets of graphene sheets, with a finite diameter denoted by $\mathrm{D}$. However, due to the very high aspect ratio of CNTs, which can be of the order of $10^{3}$ between its length and diameter, it is cumbersome to model them as cylindrical volume domains regarding meshing problems in the extremely thin cylinders. To overcome this problem, the CNTs with finite diameter are replaced by highly conducting lines. The local constitutive relationships relating $\mathrm{j}^{\mathrm{c}}$ with $\mathrm{E}^{\mathrm{c}}$ is defined in the CNTs by:

$$
j^{c}=\sigma^{c} E^{c}
$$

where $\sigma^{\mathrm{c}}$ denotes the equivalent line electrical conductivity of CNT and is dependent on the diameter D through:

$$
\sigma^{c}=\pi \frac{D^{2}}{4} S ; S=\sigma_{0}^{c} n \times n
$$

In (5), $\mathrm{n}$ is the unit tangent vector of CNT and $\sigma_{0}^{c}$ denotes electric conductivity of the CNT along n direction [17-19].

\section{B. Numerical discretization}

The electromagnetic problem (1-3) is solved in RVE with first order finite elements with periodical boundary conditions. The effective conductivity tensor is defined as:

$$
\bar{\sigma}=\frac{\partial \bar{J}(\bar{E})}{\partial \bar{E}}
$$

where $\bar{J}$ is the effective current density expressed by:

$$
\bar{J}=\frac{1}{V}\left\{\int_{V} j(x) d \Omega+\int_{\Gamma} j^{c}(x) d \Gamma\right\}
$$

and $\bar{E}$ is the effective electric field given by:

$$
E(x)=\frac{1}{V} \int_{V} E(x) d \Omega
$$

where $\mathrm{V}$ is the volume of $\Omega$.

In this model, the tunneling effect plays an important part in the electric conduction, and it is dependent on a distance $\mathrm{d}$ between CNTs.

\section{NUMERICAL RESULTS}

In the calculations, the electrical conductivities of the polymer and CNTs are taken as $\sigma^{m}=10^{-10} \mathrm{~S} / \mathrm{m}$ and $\sigma_{0}^{c}=$ $10^{6} \mathrm{~S} / \mathrm{m}$. The length of CNTs is $1=2 \mu \mathrm{m}$, and the aspect ratio $\eta=1 / \mathrm{D}$ may change with various diameters $\mathrm{D}$. The applied electric field is $\bar{E}_{i}=0.125 \mathrm{~V} / \mu \mathrm{m} \quad(\mathrm{i}=1,2,3)$. The cutoff distance of the tunneling effect is $\mathrm{d}_{\text {cutoff }}=1.7 \mathrm{~nm}$.

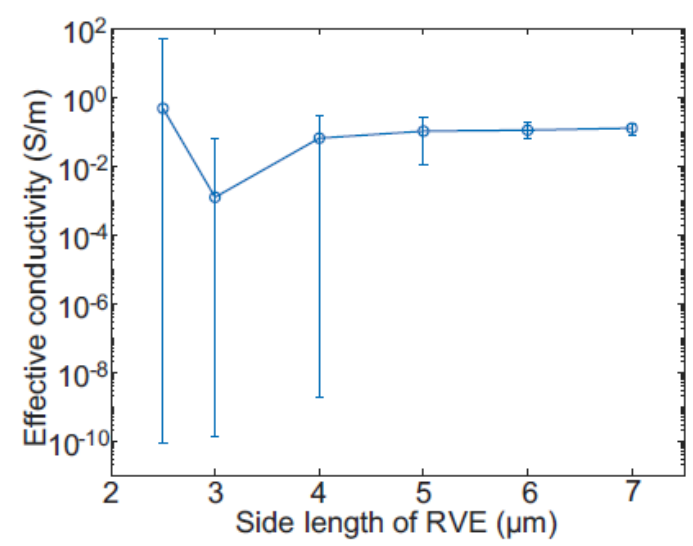

Fig. 2. Mean values and the ranges of effective conductivity versus the side length of RVE. For each case, the number of realizations insures the convergence of the mean value. The aspect ratio CNTs $=1 / \mathrm{D}=40$, the CNTs volume fraction is $2.2 \mathrm{vol} \%$, the barrier height $\phi_{0}=10 \mathrm{eV}$, and the applied electric field is $0.125 \mathrm{~V} / \mu \mathrm{m}$.

In order to determine the RVE size, the averaged effective electrical conductivity is plotted on Fig.2 and its ranges as a function of the side length of RVE. For each point, the number of random realizations is large enough to reach a statistic convergence. It shows that the dispersion of the results 
decreases when the size of the domain increases. The mean values converge when the side length of the RVE is roughly 5 $\mu \mathrm{m}$, which is the value used in the following analysis.

\section{A. Effective static conductivity}

In this section, the proposed methodology is used to investigate the influence of CNTs volume fraction on the effective electric conductivity of the composites. The volume fraction of CNTs is increased by increasing the number of introduced CNTs in the domain. Several realizations with various CNTs volume fraction ranging from 0.1 vol\% to 0.8 vol\% are investigated (Fig. 3). The aspect ratio of the CNTs is 100. The barrier height between the matrix and CNTs is taken as $\phi_{0}=10 \mathrm{eV}$. Taking into account the tunneling effect, the numerical value of $\sigma_{x}$, is plotted as a function of CNTs volume fraction in Fig. 4 where the average values are obtained for 40 realizations, and their ranges are also presented (similar behaviors are observed for the two other components $\sigma_{y}, \sigma_{z}$ ). It indicates that the electrical conductivity of CNTs nanocomposites increases with the CNTs volume fraction. It should be noted that a sharp rise of the conductivity of several orders of magnitude occurs at about $0.4 \mathrm{vol} \%$ (percolation threshold), where the mean values turn to be over $10^{-8} \mathrm{~S} / \mathrm{m}$.

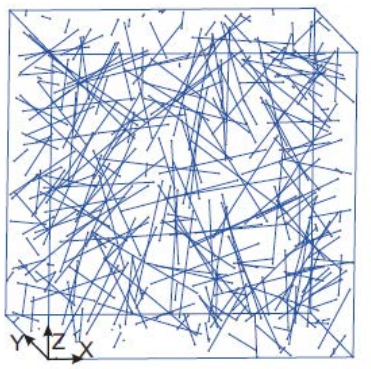

(a)

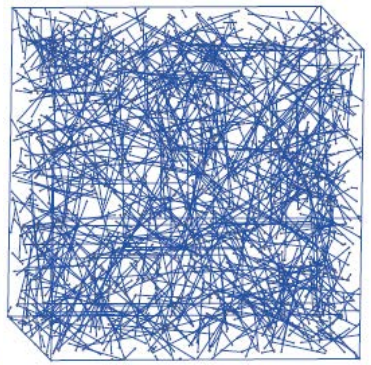

(b)
Fig. 3. Mean Realizations of microstructures for different CNT volume fractions (f), where the aspect ration $\mathrm{l} / \mathrm{D}=100 . \mathrm{f}=0.1 \%$ (a). $\mathrm{f}=0.3 \%$ (b).

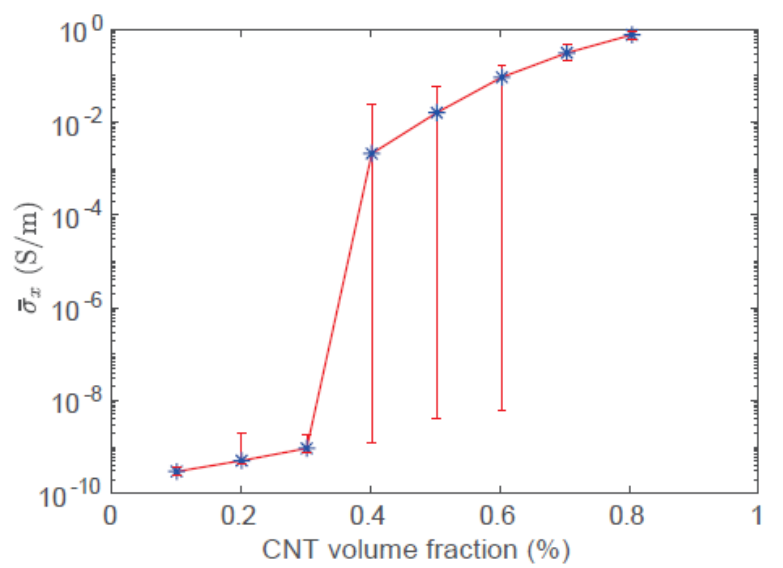

Fig. 4. Effective conductivities tensor components (mean value and range) as a function of the CNT volume fraction.

The percolation threshold is the minimum content of conductive filler in the insulating matrix which is characterized by a sharp increase in conductivity due to the formation of conductive network and realize an insulator-toconductor transition in the composites. The average values (Fig.5) are obtained for 40 realizations. The impact of the tunneling effect is very significant.

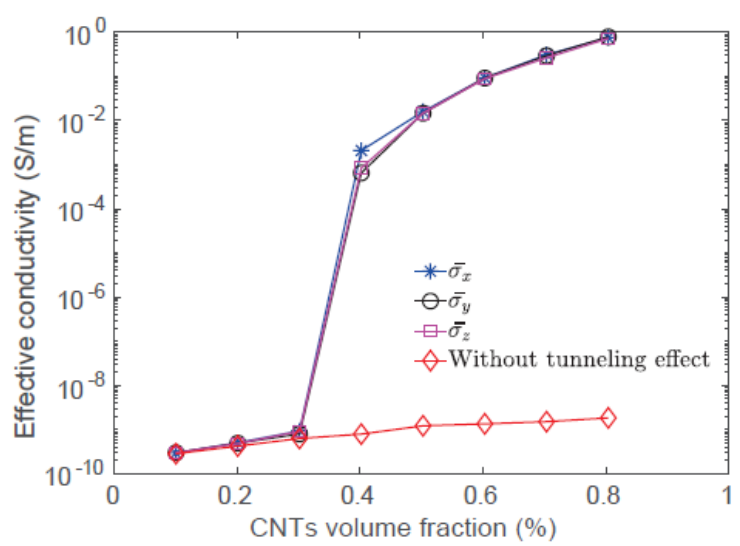

Fig. 5. Impact of the tunneling effect.

\section{B. Effective AC conductivity}

A numerical study investigating the AC conductivity and the frequency-dependent dielectric constants of $\mathrm{CNT} /$ polymer nanocomposites is summarized in this section. In the different regions of the material the current density is now expressed locally as:

$$
j=(\sigma+i \omega \epsilon) E
$$

The effective electrical conductivity tensor $\bar{\sigma}$ is defined as:

$$
\bar{\sigma}=\overline{\sigma^{\prime}}+i \overline{\sigma^{\prime \prime}}=\frac{\partial \bar{J}^{d}(\bar{E})}{\partial \bar{E}}
$$

where $\overline{J^{d}}$ and $\bar{E}$ are respectively the effective current density and effective electric field given by similar equations as in statics.

The real part of the effective conductivity in Eq. (8), corresponds to the AC conductivity measured by experimental techniques.

In this work the CNTs are randomly dispersed in the epoxy matrix. Therefore, the dielectric permittivity is assumed to be a scalar, i.e., the samples are isotropic. The measurements of the dielectric properties of the samples were conducted using three different procedures working in different frequency ranges. Measurements were conducted in a coaxial transmission line with connector (APC7 mm line) [20]. The sample is between a coaxial waveguide and a shortcircuit. The thickness of the sample is around $1 \mathrm{~mm}$ and its diameter is equal to $7 \mathrm{~mm}$. The complex permittivity and the AC conductivity are computed with an analytical method from the reflection coefficient measured [20]. Fig. 6 shows the variation of electric conductivity as a function of the frequency for neat epoxy and the nanocomposites containing various CNT contents. Experimental results are denoted by marks. For pure epoxy and the composites below the percolation threshold, the electric conductivity is frequencydependent and increases linearly with frequency in log-log scale. Beyond the percolation threshold, the CNT/polymer nanocomposite appears to be frequency-independent at frequencies below $10^{4} \mathrm{~Hz}$, followed by a region of linearly increasing conductivity along with the conductivity and the frequency in logarithmic scale. The corresponding numerical estimations are presented in Fig. 4 as comparison, where 10 random realizations are computed for each CNT content.

Below percolation threshold, each curve is computed by taking the average value of the samples due to their extremely small deviation, and the color region represents the interval of these samples for the nanocomposites with high CNT 
volume fraction above percolation threshold. It can be seen that around percolation threshold ( $0.17 \mathrm{vol} \%)$, the electrical conductivities present a very large deviation at low frequency. The numerical results are in good agreement with the obtained experimental data for the composites beyond percolation threshold. As for the pure epoxy and the composites with 0.03 vol\% CNT, the estimated electrical conductivities are higher than the experimental measurements at low frequency range below $10^{3} \mathrm{~Hz}$. The reason is that the electrical conductivity of the pure epoxy has been assumed to be $10^{-10} \mathrm{~S} / \mathrm{m}$, which is $10^{3}$ higher than the real value due to the computational limitations. It has been verified that this assumption does not influence the electrical conductivity of the composites whose CNT content is beyond percolation threshold according to the formation of current paths by highly conductive CNTs. However, with extremely low CNT volume fraction, the epoxy matrix plays important role in the effective electric conductivity at low frequency, leading to the small disagreement between numerical estimations and experimental data.

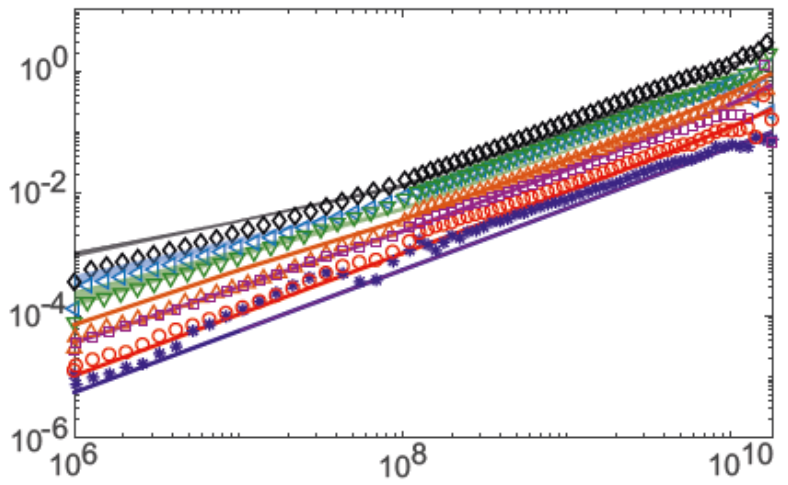

a)

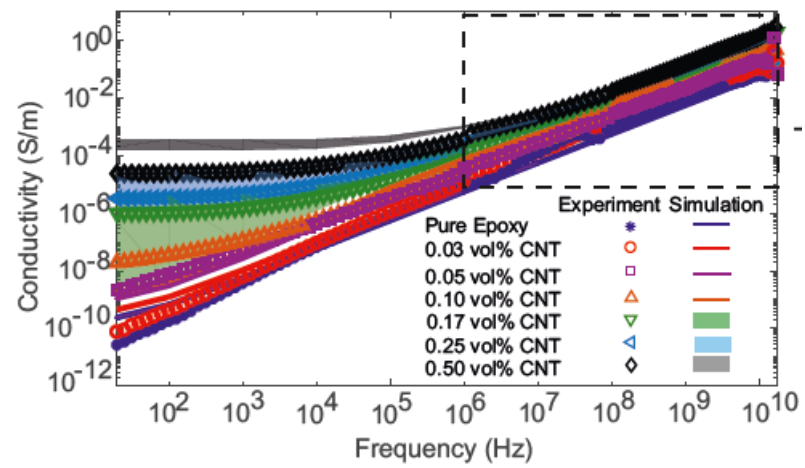

b)

Fig. 6. AC conductivity versus frequency of CNT/epoxy nanocomposites for various CNT volume fraction: broadband behavior a) and zoom b).

\section{ACKNOWLEDGMENT}

This work has benefited from the financial support of the LabEx LaSIPS (ANR-10-LABX-0032-LaSIPS) managed by the French National Research Agency under the “Investissements d'avenir” program (ANR-11-IDEX-0003).

\section{REFERENCES}

[1] B. G. Demczyk, Y. M.Wang, J. Cumings, M. Hetman, W. Han, A. Zettl, R. Ritchie, "Direct mechanical measurement of the tensile strength and elastic modulus of multiwalled carbon nanotubes", Materials Science and Engineering: A 334 (1-2), 2002, pp. 173-178.

[2] S. J. Tans, M. H. Devoret, R. J. Groeneveld, C. Dekker, Electronelectron correlations in carbon nanotubes, Nature 394 (6695), 1998 761.

[3] H. Liu, J. Gao, W. Huang, K. Dai, G. Zheng, C. Liu, C. Shen, X. Yan, J. Guo, Z. Guo, "Electrically conductive strain sensing polyurethane nanocomposites with synergistic carbon nanotubes and graphene bifillers”, Nanoscale 8 (26), 2016 pp. 12977-12989.

[4] Q. Zhang, J.-Q. Huang, W.-Z. Qian, Y.-Y. Zhang, F. Wei, “The road for nanomaterials industry: A review of carbon nanotube production, posttreatment, and bulk applications for composites and energy storage”, Small 9 (8), 2013, pp. 1237-1265.

[5] M. Martin-Gallego, M. Bernal, M. Hernandez, R. Verdejo, M. A. López- Manchado, "Comparison of filler percolation and mechanical properties in graphene and carbon nanotubes filled epoxy nanocomposites”, European Polymer Journal 49 (6), 2013, pp. 13471353.

[6] B. De Vivo et al., "Electromagnetic properties of Carbon NanoTube/epoxy nanocomposites," 2009 International Symposium on Electromagnetic Compatibility - EMC Europe, Athens, 2009, pp. 1-4.

[7] G. De Bellis, A. D'Aloia, M. S. Sarto and A. Tamburrano, "Electromagnetic modelling and experimental characterization of carbon-based nanocomposites," 2011 11th IEEE International Conference on Nanotechnology, Portland, OR, 2011, pp. 1208-1211.

[8] G. Hu, C. Zhao, S. Zhang, M. Yang, Z. Wang, "Low percolation thresholds of electrical conductivity and rheology in poly (ethylene terephthalate) through the networks of multi-walled carbon nanotubes”, Polymer 47 (1), 2006, pp. 480-488.

[9] A. Ameli, M. Nofar, C. Park, P. Pötschke, G. Rizvi, "Polypropylene/ carbon nanotube nano/microcellular structures with high dielectric permittivity, low dielectric loss, and low percolation threshold", Carbon 71, 2014, pp. 206-217.

[10] S. C. Tjong, G. Liang, S. Bao, "Electrical behavior of polypropylene/ multiwalled carbon nanotube nanocomposites with low percolation threshold”, Scripta Materialia 57 (6), 2007, pp. 461-464.

[11] Y. Yu, G. Song, L. Sun, "Determinant role of tunneling resistance in electrical conductivity of polymer composites reinforced by well dispersed carbon nanotubes", Journal of Applied Physics 108 (8) (2010) 084319.

[12] C. Li, E. T. Thostenson, T.-W. Chou, "Dominant role of tunneling resistance in the electrical conductivity of carbon nanotube-based composites”, Applied Physics Letters 91 (22), 2007, pp. 223114.

[13] W. Bao, S. Meguid, Z. Zhu, M. Meguid, "Modeling electrical conductivities of nanocomposites with aligned carbon nanotubes", Nanotechnology 22 (48), 2011, pp. 485704.

[14] W. Bao, S. Meguid, Z. Zhu, G. Weng, "Tunneling resistance and its effect on the electrical conductivity of carbon nanotube nanocomposites”, Journal of Applied Physics 111 (9), 2012, 093726.

[15] S. Gong, Z. Zhu, S. Meguid, "Anisotropic electrical conductivity of polymer composites with aligned carbon nanotubes", Polymer 56, 2015, pp. 498-506.

[16] J. G. Simmons, "Generalized formula for the electric tunnel effect between similar electrodes separated by a thin insulating film”, Journal of applied physics 34 (6), 1963, pp. 1793-1803.

[17] X. Lu, J. Yvonnet, F. Detrez, J. Bai, "Multiscale modeling of nonlinear electric conductivity in graphene-reinforced nanocomposites taking into account tunnelling effect”, Journal of Computational Physics 337, 2017, pp. 116-131.

[18] A. Bossavit, "small parameter" problems in eddy-current theory: a review, and case-study on how to avoid meshing small air-gaps", IEEE Transactions on Magnetics 32 (3), 1996, pp 729-732.

[19] J. Yvonnet, Q.-C. He, C. Toulemonde, "Numerical modelling of the effective conductivities of composites with arbitrarily shaped inclusions and highly conducting interface”, Composites Science and Technology 68 (13) (2008) 2818-2825.

[20] N.-E. Belhadj-Tahar, A. Fourrier-Lamer, "Broad-band analysis of a coaxial discontinuity used for dielectric measurements”, IEEE transactions on Microwave Theory and Techniques 34 (3), 1986, pp. 346-350. 